\title{
In memoriam Paul Ottino
}

\section{Claude Robineau}

\section{OpenEdition}

Journals

Édition électronique

URL : http://journals.openedition.org/jso/1556

DOI : 10.4000/jso.1556

ISSN : 1760-7256

\section{Éditeur}

Société des océanistes

\section{Édition imprimée}

Date de publication : 1 décembre 2002

Pagination : 229-231

ISSN : 0300-953x

\section{Référence électronique}

Claude Robineau, «In memoriam Paul Ottino », Journal de la Société des Océanistes [En ligne], 114-115 Année 2002, mis en ligne le 26 mai 2008, consulté le 22 mars 2021. URL : http:// journals.openedition.org/jso/1556 ; DOI : https://doi.org/10.4000/jso.1556

\section{(c) (1) (9)}

Journal de la société des océanistes est mis à disposition selon les termes de la Licence Creative Commons Attribution - Pas d'Utilisation Commerciale - Pas de Modification 4.0 International. 


\section{Actualités}

\section{In memoriam Paul Ottino}

par

\section{Claude ROBINEAU}

Paul Ottino, ethnologue, professeur émérite des Universités, vient de nous quitter. Né en 1930, homme de l'après-guerre, il incarnait au sortir des horreurs de la Seconde Guerre mondiale une vision volontairement optimiste du monde que les désastres économiques, militaires, écologiques accumulés et accélérés à la fin du vingtième siècle infléchirent vers un pessimisme grandissant qui n'était pas dans le dynamisme de sa nature.

Tout dans son itinéraire l'oppose aux générations de chercheurs qui ont suivi la sienne. Niçois aux origines provençale et piémontaise, il ne vient qu'assez tard à la recherche, vers la trentaine, par une route où se mêlent l'attrait du lointain et des autres, la fondation d'une famille et l'acquisition, en sus de la vie professionnelle et familiale, d'une formation universitaire qui aboutit au cumul de diplômes de doctorat: sciences économiques, troisième cycle d'anthropologie, lettres et sciences humaines (la « grande thèse »). De notre temps, on vient tard à la recherche parce qu'avant les années soixante, la filière s'en ébauche à peine dans les sciences humaines.

Madagascar est le lieu d'un premier contact continu avec des cultures différentes et deviendra le premier terrain du chercheur. C'est ici que nous nous rencontrons pour la première fois et découvrons en même temps Antananarivo, la "Ville-des-Mille», au pays des Ambaniandro, « ceux qui sont sous le soleil », lieu symbolique de rencontre entre les mondes africain et indo- malais qui renvoie aux origines du peuplement de la Grande Île, aux phénomènes d'acculturation, à la structure et à la dynamique des cultures. Trois années passées sur la côte nord-ouest forgent chez Paul un regard d'ethnologue.

Entré en 1958 à l'ORSTOM, actuel IRD, il est envoyé fortuitement à Madagascar pour y remplacer l'ethnologue Louis Molet affecté par la Maison à Tahiti. Parlant couramment le malgache, y compris les dialectes du Nord-Ouest (Analalava et Ambaja), Ottino a séduit, par son esprit d'ouverture et sa capacité à susciter le dialogue, le gouverneur Deschamps, l'ethnologue des Antaisaka alors en charge des sciences humaines à l'orstom. Paul est alors envoyé dans le SudOuest malgache pour une mission socioéconomique d'expertise pour le développement de la région du delta du Mangoky, accompagné de deux autres ethnologues, Anne et Henri Lavondès, et d'un jeune géographe, Jean-Pierre Trouchaud. Va commencer avec Lavondès un long et fructueux dialogue où le dynamisme, voire l'exubérance, du jeune chercheur vont se frotter et se polir à la rigueur du scholar, enseignant de latin-grec en Savoie qu'une enquête sur le franco-provençal parlé en cette région a converti à l'ethnologie. Cette mission "Mangoky » que Georges Condominas vient mettre en place pour l'ORSTOM - ainsi s'amorce avec Paul et Henri une relation d'une rare qualité - illustre et clôt à la fois la période économique d'Ottino avec le livre sur les Économies paysannes malgaches du Bas-Mangolg, qui est la syn- 
thèse de la part personnelle de l'enquête collective et d'un travail spécifique sur l'économie indienne ("pakistanaise ») dans le Sud-Ouest malgache. La puissance de travail de Paul est étonnante, qu'il s'agisse du " terrain » effectué à hautes doses dans la langue du pays, de la littérature scientifique qu'il dévore, de l'abondance des publications, livres et articles en anglais et en français, voire de nouvelle langues dont il fera l'apprentissage tout au long de sa vie. Paul parle excellemment l'anglais (y compris l'American English), l'italien, le malgache, le tahitien, voire l'espagnol, le portugais, l'arabe et le swahili ; il fréquente le néerlandais qu'il trouve difficile, mais les Indes néerlandaises ont été à la source d'une littérature anthropologique non négligeable, le japonais parce que le paysage naturel et urbain l'enchante, l'allemand, dont j'ai découvert sa connaissance avec cette langue il n'y a pas six mois, et de me conseiller, avec sa persuasion coutumière, tel merveilleux manuel d'apprentissage oral - legs dernier qui m'émeut.

Mais pendant sa période économique, Paul m'entretenait épistolairement depuis son lointain Mangoky, des structures sociales effervescentes et des formes de sociabilité, une passe intermédiaire gurvitchienne qui allait le mener rapidement par continuité-rupture à Claude Lévi-Strauss. Le temps de l'ethnologie ottinienne primitive, où l'on allait contempler de concert les vitrines déjà un peu ternes du Musée de l'Homme (puisse le temple promis du quai Branly être aussi pédagogique et sans prétention que son ancêtre du Trocadéro) avait fait place à une fureur d'anthropologie britannique et américaine, d'Evans-Pritchard à Murdock et à Leach, en passant par Radcliffe-Brown, les African Political Systems et les ouvrages de Raymond Firth. Cela a évidemment duré jusqu'au terrain polynésien et au delà...

Pendant ce temps, les travaux malgaches ont continué: Sakay sur les plateaux que Paul n'aimait pas trop, Anony dans la dépression intermédiaire entre les deux falaises orientales. Il s'agit là d'analyses de structures sociales en vue du développement de ces régions, l'une dans le Moyen-Ouest, l'autre aux abords du lac Alaotra. Paul est beaucoup plus a l'aise aux prises, sur l'Alaotra, avec des dominantes merina que dans l'étude de la parenté sur la Sakay où il se trouve pourtant là avec Lavondès. Sur ce terrain, tous deux ont procédé à une analyse rigoureuse notamment inspirée de Louis Dumont, qui vient à la suite de celle qu'a engagée Lavondès sur le terrain de Mangoky. La relecture de ce rapport, malheureusement non publié, sur les structures de la Sakay, montrant la liaison entre la règle merina de la société et de la terre avec, notamment, l'accent mis sur la non-dispersion de l'héritage en cas de succession, révèle que les chercheurs n'étaient pas loin de la clé du système merina de parenté fondé sur le clan endogame accroché à la terre des ancêtres. Ottino est véritablement entré dans la grande période anthropologique qu'il ne quittera plus, mêlant terrain et enseignement à Nanterre, à l'EHESS, à la Réunion.

Vient, à la fin de 1962, le premier et le grand séjour polynésien. Le premier travail de Paul est consacré à la pêche au grand filet à Tautira, à l'extrémité nord-est de Tahiti-iti, qui lui fait découvrir la figure d'un vrai leader polynésien. Sujet de recherche moins anodin, voire décevant, qu'il n'y paraît car la figure du leader est la clé de la sociologie polynésienne ancienne et actuelle. Paul signale en outre, dans ce travail, le rôle des factions à l'œuvre dans la société de Tautira, alignement pour ou contre le leader des maisonnées du village en fonction des liens de parenté ou professionnels, des appartenances de milieux sociaux, religieuses, politiques, des conflits de personnes, de familles, de terres : alignement qui a sa correspondance dans les réseaux adverses qui, à un moment donné, configurent socialement l'île entière, voire le Territoire, y faisant apparaître une structure dualiste.

Un second travail, Ethnohistoire de Rangiroa, repris dans un livre d'hommage à l'archéologue américain Kenneth Emory ("Early 'ati of the Western Tuamotu ») s'efforce de retrouver, dans un atoll influencé par la culture ma'ohi, l'existence de groupements lignagers anciens 'ati, groupes de parents liés à la terre et à des structures lithiques de caractère religieux appelés en Polynésie orientale marae, et il établit ce qu'aux îles de la Société affirmait la tradition orale, sans qu'on puisse le vérifier par des exemples certains, la correspondance entre marae, 'ati et terre nommément désignée. Cette Ethno-Histoire est le premier livre d'une grande œuvre à venir dont une " ethno-économie », qui ne verra jamais le jour, devait être le second, car Paul, lancé dans un inventaire de maisonnées et de terres, et dans le recueil de généalogies, se trouve maintenant engagé au cœur de l'anthropologie sociale la plus classique avec la recherche des règles de filiation et d'alliance ainsi que des droits sur les terres et leur dévolution successorale. La maîtrise de la théorie de la parenté, les acquis malgaches et la formation première de juriste produisent Rangiroa: parenté étendue, résidence et terre dans un atoll polynésien, sa grande thèse pour le doctorat ès lettres qui va lui ouvrir, à terme, un professorat que lui, homme de terrain, n'a pas recherché 
d'emblée, mais auquel il accède après avoir quitté l'ORSTOM.

Rangiroa apporte une vue neuve sur la parenté en Polynésie orientale, avec l'accent mis dans la parentèle (les feti'i) sur le groupe des frères et sœurs ('opu ho'e), élément pertinent qui structure l'organisation sociale, les relations de génération ayant le pas sur celles de filiation, ou plutôt ces derniers, ainsi que les alliances, devant être analysés comme des filiations et des alliances de 'opu ho'e. Cette notion de pertinence était très importante dans la sociologie et l'anthropologie de Paul qui, a contrario, qualifiait d' " irrelevant » ce qui ne lui apparaissait pas pertinent à l'enquête ou à l'analyse. Il souligne également, dans les relations sociales ma'ohi, la fonction de pattern exercée par les feti' $i$, de la parenté comme modèle de ces dernières. Enfin, et ce n'est pas le moindre des apports de son livre, il montre - et c'était déjà en germe dans sa pensée dans « Early 'ati »- que, dans ce système de parenté indifférenciée, c'est la relation à la terre qui permet, à chaque génération, le choix entre les lignées paternelle et maternelle, le principe d'indifférenciation obligeant, dans un système de parenté lignagère exclusive, à l'introduction d'un élément de surdétermination, ici la résidence et la terre. Toutes ces notions qui font partie du corpus de connaissances actuelles à Tahiti concernant la société, c'est à Ottino qu'on doit de les avoir rendues patentes. Et il est arrivé à Paul Ottino, comme avant lui à Louis Dumont, d'être mieux connu et apprécié pour ses travaux dans le monde anglo-saxon, notamment aux ÉtatsUnis, qu'en France où il demeure en marge du main stream sociologique, plus tenu sur la touche, d'ailleurs, qu'ignoré.
Vient donc le temps de l'enseignement et de la maturation. Professeur associé à Nanterre (Paris $\mathrm{X})$. Directeur d'études à l'EHESS, où il œuvre de concert avec l'historienne et malgachisante Françoise Raison-Jourde. Professeur à l'Université de la Réunion où il fonde une École doctorale en ethnologie qu'il anime de longues années. Époque d'enseignement où il excellait, passionnant les étudiants, entrecoupée de séjours de terrain, particulièrement à Madagascar. Les travaux de ces années enseignantes manifestent alors sa maîtrise de la discipline, que ce soit dans sa méthode d'ethnologie réflexive qu'il qualifie d'«anthropologie généralisée » ou dans le domaine de l'histoire culturelle du Pacifique et de l'Océan indien où il se complaît, qu'il s'agisse de L'étrangère intime, essai de civilisation de l'Ancien Madagascar (1986), de «L'empreinte culturelle musulmane dans l'Océan indien » (publié dans l'Encyclopédie philosophique universelle) ou des Champs de l'ancestralité à Madagascar, parentés alliance et patrimoine (1998), son dernier livre auquel il tient beaucoup et qui referme la boucle malgache ouverte quarantecinq années plus tôt.

On se souviendra de Paul Ottino comme d'un être dynamique, un chercheur passionné et passionnant, un enseignant toujours chaleureux, mais exigeant, gros travailleur toujours sur la brèche, qui prenait son plaisir dans la vie en brousse, la pêche sous-marine et l'apprentissage des langues vivantes. Il me plaît qu'il ait voulu reposer à Moorea, au bord de la baie d'Opunohu, dans cette Polynésie qu'il chérissait, dont il aimait la simplicité et où il se fit, parmi ses habitants, de très fidèles amis. 